\title{
On the improvement of Mocanu's conditions
}

\author{
M Nunokawa' ${ }^{1}$, S Owa $^{2}$, NE Cho ${ }^{3 *}$, J Sokół ${ }^{4}$ and E Yavuz Duman ${ }^{5}$
}

${ }^{*}$ Correspondence:
necho@pknu.ac.kr
${ }^{3}$ Pukyong National University,
Pusan, 608-737, Korea
Full list of author information is
available at the end of the article

\begin{abstract}
We estimate $|\operatorname{Arg}\{p(z)\}|$ for functions of the form $p(z)=1+a_{1} z+a_{2} z^{2}+a_{3} z^{3}+\cdots$ in the unit disc $\mathbb{D}=\{z:|z|<1\}$ under several assumptions. By using Nunokawa's lemma, we improve a few of Mocanu's results obtained by differential subordinations. Some applications for strongly starlikeness and convexity are formulated.
\end{abstract}

MSC: Primary 30C45; secondary 30C80

Keywords: Nunokawa's lemma; strongly starlike functions of order alpha; strongly convex functions of order alpha; subordination

\section{Introduction}

Let $\mathcal{H}$ be the class of functions analytic in the unit disk $\mathbb{D}=\{z \in \mathbb{C}:|z|<1\}$, and denote by $\mathcal{A}$ the class of analytic functions in $\mathbb{D}$ and usually normalized, i.e., $\mathcal{A}=\{f \in \mathcal{H}: f(0)=$ $\left.0, f^{\prime}(0)=1\right\}$.

Let $\mathcal{S S}^{*}(\beta)$ denote the class of strongly starlike functions of order $\beta, 0<\beta \leq 1$,

$$
\mathcal{S S}^{*}(\beta):=\left\{f \in \mathcal{A}:\left|\operatorname{Arg} \frac{z f^{\prime}(z)}{f(z)}\right|<\frac{\beta \pi}{2}, z \in \mathbb{D}\right\}
$$

which was introduced in [1] and [2]. We say that $f \in \mathcal{A}$ is in the class $\mathcal{S C}^{*}(\beta)$ of strongly convex functions of order $\beta$ when $z f^{\prime}(z) \in \mathcal{S S}^{*}(\beta)$. We say that $f \in \mathcal{H}$ is subordinate to $g \in \mathcal{H}$ in the unit disc $\mathbb{D}$, written $f \prec g$ if and only if there exists an analytic function $w \in$ $\mathcal{H}$ such that $w(0)=0,|w(z)|<1$ and $f(z)=g[w(z)]$ for $z \in \mathbb{D} \subseteq g(\mathbb{D})$. In particular, if $g$ is univalent in $\mathbb{D}$ then the subordination principle says that $f \prec g$ if and only if $f(0)=g(0)$ and $f(|z|<r) \subseteq g(|z|<r)$ for all $r \in(0,1)$.

\section{Main result}

In this section, we investigate conditions, under which a function $f \in \mathcal{A}$ is strongly starlike or strongly convex. We also estimate $|\operatorname{Arg}\{p(z)\}|$ for functions of the form $p(z)=$ $1+a_{1} z+a_{2} z^{2}+a_{3} z^{3}+\cdots$ in the unit disc $\mathbb{D}$, under several assumptions, and then we use this estimation for the case $p(z)=z f^{\prime}(z) / f(z)$. By using Nunokawa's lemma [3], we improve a few Mocanu's $[4,5]$ results obtained by differential subordinations. Some sufficient conditions for functions to be in several subclasses of strongly starlike functions can also be found in the recent papers [6] and [7-11].

Theorem 2.1 Let $f(z)=z+\sum_{n=2}^{\infty} a_{n} z^{n}$ be analytic in the unit disc $\mathbb{D}$. If

$$
\left|\operatorname{Arg}\left\{f^{\prime}(z)\right\}\right|<\frac{\alpha \pi}{2} \approx 1.0076658, \quad z \in \mathbb{D}
$$


where $\alpha=1 /(1+\beta)=1 /(2-(\log 4) / \pi) \approx 0.641548, \beta=1-(\log 4) / \pi \approx 0.5587$, then

$$
\left|\operatorname{Arg}\left\{\frac{z f^{\prime}(z)}{f(z)}\right\}\right|<\frac{\pi}{2}, \quad z \in \mathbb{D}
$$

orf is starlike in $\mathbb{D}$.

Proof By (2.1), we have

$$
\left\{f^{\prime}(z)\right\}^{1 / \alpha} \prec \frac{1+z}{1-z}, \quad z \in \mathbb{D}
$$

Let $z=\rho e^{i \theta}, \rho \in[0,1), \theta \in(-\pi, \pi]$. The function $w(z)=(1+z) /(1-z)$ is univalent in $\mathbb{D}$ and maps $|z|<\rho<1$ onto the open disc $D(C, R)$ with the center $C=\left(1+\rho^{2}\right) /\left(1-\rho^{2}\right)$ and the radius $R=(2 \rho) /\left(1-\rho^{2}\right)$. Then by the subordination principle under univalent function,

$$
\left\{f^{\prime}\left(x e^{i \theta}\right)\right\}^{1 / \alpha} \in D(C, R) \quad \text { for all } x \in[0, \rho), \theta \in(-\pi, \pi]
$$

A simple geometric observation yields to

$$
\left|\operatorname{Arg}\left\{\left(f^{\prime}\left(\rho e^{i \theta}\right)\right)^{1 / \alpha}\right\}\right| \leq \sin ^{-1} \frac{R}{C}=\sin ^{-1} \frac{2 \rho}{1+\rho^{2}} \quad \text { for all } \rho \in[0,1), \theta \in(-\pi, \pi] \text {. }
$$

Therefore, applying the same idea as [3, pp.1292-1293] for $z=r e^{i \theta}, r \in[0,1), \theta \in(-\pi, \pi]$, we have

$$
\begin{aligned}
\left|\operatorname{Arg}\left\{\frac{f(z)}{z}\right\}\right| & =\left|\operatorname{Arg}\left\{\int_{0}^{r} f^{\prime}\left(\rho e^{i \theta}\right) \mathrm{d} \rho\right\}\right| \\
& \leq \int_{0}^{r}\left|\operatorname{Arg}\left\{f^{\prime}\left(\rho e^{i \theta}\right)\right\}\right| \mathrm{d} \rho \\
& =\alpha \int_{0}^{r}\left|\operatorname{Arg}\left\{\left(f^{\prime}\left(\rho e^{i \theta}\right)\right)^{1 / \alpha}\right\}\right| \mathrm{d} \rho \\
& \leq \alpha \int_{0}^{r} \sin ^{-1} \frac{2 \rho}{1+\rho^{2}} \mathrm{~d} \rho \\
& =\left.\alpha\left\{\rho \sin ^{-1} \frac{2 \rho}{1+\rho^{2}}-\log \left(1+\rho^{2}\right)\right\}\right|_{\rho=0} ^{\rho=r} \\
& =\alpha\left\{r \sin ^{-1} \frac{2 r}{1+r^{2}}-\log \left(1+r^{2}\right)\right\} .
\end{aligned}
$$

The function

$$
h(r)=r \sin ^{-1} \frac{2 r}{1+r^{2}}-\log \left(1+r^{2}\right), \quad r \in[0,1)
$$

is increasing because $h^{\prime}(r)=\sin ^{-1}\left\{2 r /\left(1+r^{2}\right)\right\}>0$. Now, letting $r \rightarrow 1^{-}$, we obtain

$$
\begin{aligned}
\left|\operatorname{Arg}\left\{\frac{f(z)}{z}\right\}\right| & \leq \alpha(\pi / 2-\log 2)=\frac{\alpha \pi}{2}\left\{1-\frac{\log 4}{\pi}\right\} \\
& =\frac{\pi}{2} \alpha \beta, \quad z \in \mathbb{D} .
\end{aligned}
$$


Using this and (2.1), we obtain

$$
\begin{aligned}
\left|\operatorname{Arg}\left\{\frac{z f^{\prime}(z)}{f(z)}\right\}\right| & \leq\left|\operatorname{Arg}\left\{f^{\prime}(z)\right\}\right|+\left|\operatorname{Arg}\left\{\frac{f(z)}{z}\right\}\right| \\
& <\frac{\alpha \pi}{2}+\frac{\pi}{2} \alpha \beta \\
& =\frac{\alpha(1+\beta) \pi}{2} \\
& =\frac{\pi}{2}, \quad z \in \mathbb{D} .
\end{aligned}
$$

It completes the proof.

Remark 2.2 Theorem 2.1 is an improvement of Mocanu's result in [4].

Theorem 2.3 Let $p(z)=1+\sum_{n=1}^{\infty} a_{n} z^{n}$ be analytic in the unit disc $\mathbb{D}$. If

$$
\mathfrak{R e}\left\{p(z)+z p^{\prime}(z)\right\}>0, \quad z \in \mathbb{D}
$$

then

$$
|\operatorname{Arg}\{p(z)\}|<\frac{\pi}{2}-\log 2=0.877649 \ldots, \quad z \in \mathbb{D}
$$

Proof By (2.5), we have

$$
p(z)+z p^{\prime}(z) \prec \frac{1+z}{1-z}, \quad z \in \mathbb{D} .
$$

Let $z=\rho e^{i \theta}, \rho \in[0,1), \theta \in(-\pi, \pi]$. The subordination principle used for (2.7) gives

$$
\left|p\left(x e^{i \theta}\right)+x e^{i \theta} p^{\prime}\left(x e^{i \theta}\right)-\frac{1+\rho^{2}}{1-\rho^{2}}\right|<\frac{2 \rho}{1-\rho^{2}} \quad \text { for all } x \in[0, \rho), \theta \in(-\pi, \pi] .
$$

A simple geometric observation yields to

$$
\left|\operatorname{Arg}\left\{p\left(\rho e^{i \theta}\right)+\rho e^{i \theta} p^{\prime}\left(\rho e^{i \theta}\right)\right\}\right| \leq \sin ^{-1} \frac{2 \rho}{1+\rho^{2}} \quad \text { for all } \rho \in[0,1), \theta \in(-\pi, \pi]
$$


Therefore, for $z=r e^{i \theta}, r \in[0,1), \theta \in(-\pi, \pi]$, we have

$$
\begin{aligned}
|\operatorname{Arg}\{p(z)\}| & =\left|\operatorname{Arg}\left\{\frac{z p(z)}{z}\right\}\right| \\
& =\left|\operatorname{Arg}\left\{\frac{\int_{0}^{z}(t p(t))^{\prime} \mathrm{d} t}{z}\right\}\right| \\
& =\left|\operatorname{Arg}\left\{\frac{\int_{0}^{z}\left(p(t)+t p^{\prime}(t)\right) \mathrm{d} t}{z}\right\}\right| \\
& =\left|\operatorname{Arg}\left\{\frac{\int_{0}^{r}\left(p\left(\rho e^{i \theta}\right)+\rho e^{i \theta} p^{\prime}\left(\rho e^{i \theta}\right)\right) e^{i \theta} \mathrm{d} \rho}{r e^{i \theta}}\right\}\right| \\
& =\left|\operatorname{Arg}\left\{\int_{0}^{r}\left(p\left(\rho e^{i \theta}\right)+\rho e^{i \theta} p^{\prime}\left(\rho e^{i \theta}\right)\right) \mathrm{d} \rho\right\}-\operatorname{Arg}\{r\}\right| \\
& \leq \int_{0}^{r}\left|\operatorname{Arg}\left\{p\left(\rho e^{i \theta}\right)+\rho e^{i \theta} p^{\prime}\left(\rho e^{i \theta}\right)\right\}\right| \mathrm{d} \rho .
\end{aligned}
$$

Therefore, by using (2.9), we have

$$
\begin{aligned}
|\operatorname{Arg}\{p(z)\}| & \leq \alpha \int_{0}^{r} \sin ^{-1} \frac{2 \rho}{1+\rho^{2}} \mathrm{~d} \rho \\
& =\left.\alpha\left\{\rho \sin ^{-1} \frac{2 \rho}{1+\rho^{2}}-\log \left(1+\rho^{2}\right)\right\}\right|_{\rho=0} ^{\rho=r} \\
& <\left.\alpha\left\{\rho \sin ^{-1} \frac{2 \rho}{1+\rho^{2}}-\log \left(1+\rho^{2}\right)\right\}\right|_{\rho=0} ^{\rho=1} \\
& =\frac{\pi}{2}-\log 2=0.8776491464 \ldots, \quad z \in \mathbb{D} .
\end{aligned}
$$

It leads to the desired conclusion.

Remark 2.4 Theorem 2.3 is an improvement of Mocanu's result in [5], where instead of $\gamma_{0}=\frac{\pi}{2}-\log 2=0.8776491464 \ldots$ is

$$
\theta_{1}=\max \left\{\theta:\left|\operatorname{Arg}\left\{\frac{2}{e^{i \theta}} \log \left(1+e^{i \theta}\right)-1\right\}\right|\right\}=0.91106219 \ldots
$$

Substituting $p(z)=f(z) / z, f \in \mathcal{A}$, in Theorem 2.3 leads to the following corollary.

Corollary 2.5 If $f \in \mathcal{A}$ and it satisfies

$$
\mathfrak{R e}\left\{f^{\prime}(z)\right\}>0, \quad z \in \mathbb{D},
$$

then

$$
\left|\operatorname{Arg}\left\{\frac{f(z)}{z}\right\}\right|<\frac{\pi}{2}-\log 2=0.877649 \ldots, \quad z \in \mathbb{D} .
$$

Substituting $p(z)=z f^{\prime}(z) / f(z), f \in \mathcal{A}$, in Theorem 2.3 gives the following corollary. 
Corollary 2.6 If $f \in \mathcal{A}$ and it satisfies

$$
\mathfrak{R e}\left\{\frac{z f^{\prime}(z)}{f(z)}\left(2+\frac{z f^{\prime \prime}(z)}{f^{\prime}(z)}\right)-\left(\frac{z f^{\prime}(z)}{f(z)}\right)^{2}\right\}>0, \quad z \in \mathbb{D}
$$

then

$$
\left|\operatorname{Arg}\left\{\frac{z f^{\prime}(z)}{f(z)}\right\}\right|<\frac{\pi}{2}-\log 2=0.877649 \ldots, \quad z \in \mathbb{D} .
$$

This means that $f$ is strongly starlike of order $1-(\log 4) / \pi=0.558728799 \ldots$.

Substituting $p(z)=1+z f^{\prime \prime}(z) / f^{\prime}(z), f \in \mathcal{A}$, in Theorem 2.3 gives the following corollary.

Corollary 2.7 If $\in \mathcal{A}$ and it satisfies

$$
\mathfrak{R e}\left\{1+\frac{z f^{\prime \prime}(z)}{f^{\prime}(z)}\left(2+\frac{z f^{\prime \prime \prime}(z)}{f^{\prime \prime}(z)}\right)-\left(\frac{z f^{\prime \prime}(z)}{f^{\prime}(z)}\right)^{2}\right\}>0, \quad z \in \mathbb{D}
$$

then

$$
\left|\operatorname{Arg}\left\{1+\frac{z f^{\prime \prime}(z)}{f^{\prime}(z)}\right\}\right|<\frac{\pi}{2}-\log 2=0.877649 \ldots, \quad z \in \mathbb{D} .
$$

This means that $f$ is strongly convex of order $1-(\log 4) / \pi=0.558728799 \ldots$.

Theorem 2.8 Let $f(z)=z+\sum_{n=2}^{\infty} a_{n} z^{n}$ be analytic in the unit disc $\mathbb{D}$, and suppose that

$$
\left|f^{\prime}(z)-1\right|<1, \quad z \in \mathbb{D}
$$

Then we have

$$
\left|\operatorname{Arg}\left\{\frac{z f^{\prime}(z)}{f(z)}\right\}\right|<(1+r) \sin ^{-1} r+\sqrt{1-r^{2}}-1
$$

where $r=|z|<1$, and, therefore, we have

$$
\mathfrak{R e}\left\{\frac{z f^{\prime}(z)}{f(z)}\right\}>0 \quad \text { for }|z|<r_{0}
$$

where $0.902<r_{0}<0.903$ is the positive root of the equation

$$
\sin ^{-1} r=\frac{\pi-2\left(\sqrt{1-r^{2}}-1\right)}{2(1+r)} .
$$

Proof From (2.10), we have $f^{\prime}(z) \prec 1+z$, so the subordination principle gives

$$
\left|\operatorname{Arg}\left\{f^{\prime}(z)\right\}\right| \leq \sin ^{-1}|z|, \quad z \in \mathbb{D}
$$


and for $z=r e^{i \theta}$,

$$
\begin{aligned}
\left|\operatorname{Arg}\left\{\frac{f(z)}{z}\right\}\right| & =\left|\operatorname{Arg}\left\{\frac{1}{r e^{i \theta}} \int_{0}^{r} f^{\prime}\left(\rho e^{i \theta}\right) e^{i \theta} \mathrm{d} \rho\right\}\right| \\
& =\left|\operatorname{Arg}\left\{\int_{0}^{r} f^{\prime}\left(\rho e^{i \theta}\right) \mathrm{d} \rho\right\}\right| \\
& \leq \int_{0}^{r}\left|\operatorname{Arg}\left\{f^{\prime}\left(\rho e^{i \theta}\right)\right\}\right| \mathrm{d} \rho \\
& <\int_{0}^{r} \sin ^{-1} \rho \mathrm{d} \rho .
\end{aligned}
$$

Then we have

$$
\int_{0}^{r} \sin ^{-1} \rho \mathrm{d} \rho=r \sin ^{-1} r+\sqrt{1-r^{2}}-1
$$

Therefore, and from (2.14), we have

$$
\begin{aligned}
& \left|\operatorname{Arg}\left\{\frac{z f^{\prime}(z)}{f(z)}\right\}\right| \\
& \quad \leq\left|\operatorname{Arg}\left\{f^{\prime}(z)\right\}\right|+\left|\operatorname{Arg}\left\{\frac{f(z)}{z}\right\}\right| \\
& \quad<\sin ^{-1} r+r \sin ^{-1} r+\sqrt{1-r^{2}}-1, \quad|z|=r<1 .
\end{aligned}
$$

The function

$$
\begin{aligned}
G(r) & =(1+r) \sin ^{-1} r+\sqrt{1-r^{2}}-1 \\
& =\sin ^{-1} r+\int_{0}^{r} \sin ^{-1} \rho \mathrm{d} \rho
\end{aligned}
$$

increases in $[0,1]$ as the sum of two increasing functions. Moreover, $G(0)=0, G(1)=\pi-1$, and it satisfies

$$
G(0.902)=1.57030 \ldots<\frac{\pi}{2}=1.5707963 \ldots<G(0.903)=1.573753 \ldots
$$

Therefore, the equation (2.13) has the solution $r_{0}, 0.902<r_{0}<0.903$, and

$$
\mathfrak{R e}\left\{\frac{z f^{\prime}(z)}{f(z)}\right\}>0 \quad \text { for }|z|<r_{0} \approx 0.903
$$

This completes the proof.

Theorem 2.9 Let $f(z)=z+\sum_{n=2}^{\infty} a_{n} z^{n}$ be analytic in the unit disc $\mathbb{D}$, and suppose that

$$
\left|f^{\prime}(z)-1\right|<\alpha, \quad z \in \mathbb{D}
$$


with $\alpha \in(0,2 / \sqrt{5}]$. Then $f$ is strongly starlike of order $\beta$, where $\beta \in(0,1]$ is the positive root of the equation

$$
\sin ^{-1}\left\{\alpha \sqrt{1-\alpha^{2} / 4}+\frac{\alpha}{2} \sqrt{1-\alpha^{2}}\right\}=\frac{\pi \beta}{2} .
$$

Proof We have $f^{\prime}(z) \prec 1+\alpha z$. Applying the result from [4, p.118] we have also that $f(z) / z \prec$ $1+\alpha z / 2$ in $\mathbb{D}$. This shows that

$$
\left|\operatorname{Arg}\left\{f^{\prime}(z)\right\}\right| \leq \sin ^{-1} \alpha|z|, \quad z \in \mathbb{D}
$$

and

$$
\left|\operatorname{Arg}\left\{\frac{f(z)}{z}\right\}\right| \leq \sin ^{-1} \frac{\alpha|z|}{2}, \quad z \in \mathbb{D}
$$

Therefore, using (2.17) and (2.18), we have

$$
\begin{aligned}
& \left|\operatorname{Arg}\left\{\frac{z f^{\prime}(z)}{f(z)}\right\}\right| \\
& \quad \leq\left|\operatorname{Arg}\left\{f^{\prime}(z)\right\}\right|+\left|\operatorname{Arg}\left\{\frac{f(z)}{z}\right\}\right| \\
& <\sin ^{-1} \alpha+\sin ^{-1} \frac{\alpha}{2}, \quad z \in \mathbb{D} .
\end{aligned}
$$

For $\alpha \in(0,2 / \sqrt{5}]$, we have $\alpha^{2}+(\alpha / 2)^{2} \leq 1$, so we can use the formula

$$
\sin ^{-1} \alpha+\sin ^{-1} \frac{\alpha}{2}=\sin ^{-1}\left\{\alpha \sqrt{1-\alpha^{2} / 4}+\frac{\alpha}{2} \sqrt{1-\alpha^{2}}\right\} .
$$

The function

$$
\begin{aligned}
H(\alpha) & =\sin ^{-1}\left\{\alpha \sqrt{1-\alpha^{2} / 4}+\frac{\alpha}{2} \sqrt{1-\alpha^{2}}\right\} \\
& =\sin ^{-1} \alpha+\sin ^{-1} \frac{\alpha}{2}
\end{aligned}
$$

increases in the segment $[0,2 / \sqrt{5}]$ as the sum of two increasing functions. Moreover, $H(0)=0, H(2 / \sqrt{5})=\pi / 2$, so the equation $(2.16)$ has in $(0,1]$ the solution $\beta$. This completes the proof.

Putting $\alpha=2 / \sqrt{5}$, we get $\beta=1$ and Theorem 2.15 becomes the result from [4, p.118]:

$$
\left[\left|f^{\prime}(z)-1\right|<2 / \sqrt{5}, z \in \mathbb{D}\right] \Rightarrow\left[\mathfrak{R e}\left\{z f^{\prime}(z) / f(z)\right\}>0, z \in \mathbb{D}\right] .
$$

Theorem 2.10 Let $p(z)=1+\sum_{n=1}^{\infty} c_{n} z^{n}$ be analytic in the unit disc $\mathbb{D}$, and suppose that

$$
\left|\operatorname{Arg}\left\{p(z)+\alpha\left(\frac{z p^{\prime}(z)}{p(z)}\right)\right\}\right|<\tan ^{-1} \frac{|\alpha| \delta(\beta) \sin ((1+\beta) \pi / 2)}{1+|\alpha| \delta(\beta) \cos ((1+\beta) \pi / 2)}-\frac{\pi \beta}{2}, \quad z \in \mathbb{D},
$$


where $\alpha<0,0<\beta<1$, and

$$
\delta(\beta)=\frac{\beta}{2}\left(\left(\frac{1-\beta}{1+\beta}\right)^{\beta+1}+\left(\frac{1-\beta}{1+\beta}\right)^{\beta-1}\right) \text { and }|\alpha|>\frac{\sin (\pi \beta / 2)}{\delta(\beta)} .
$$

Then $\operatorname{Arg}\{p(z)\}<\frac{\beta \pi}{2}$ in $\mathbb{D}$.

Proof Suppose that there exists a point $z_{0} \in \mathbb{D}$ such that

$$
|\operatorname{Arg}\{p(z)\}|<\frac{\pi \beta}{2} \quad \text { for }|z|<\left|z_{0}\right|
$$

and

$$
\left|\operatorname{Arg}\left\{p\left(z_{0}\right)\right\}\right|=\frac{\pi \beta}{2}
$$

then by Nunokawa's lemma [12], we have

$$
\left\{p\left(z_{0}\right)\right\}^{1 / \beta}= \pm i a, \quad a>0 \quad \text { and } \quad \frac{z_{0} p^{\prime}\left(z_{0}\right)}{p\left(z_{0}\right)}=i k \beta,
$$

where

$$
k \geq \frac{1}{2}\left(a+\frac{1}{a}\right) \text { when } \operatorname{Arg}\left\{p\left(z_{0}\right)\right\}=\frac{\pi \beta}{2}
$$

and

$$
k \leq-\frac{1}{2}\left(a+\frac{1}{a}\right) \text { when } \operatorname{Arg}\left\{p\left(z_{0}\right)\right\}=-\frac{\pi \beta}{2},
$$

moreover,

$$
\frac{\beta k}{a^{\beta}} \geq \delta(\beta) .
$$

For the case $\operatorname{Arg}\left\{p\left(z_{0}\right)\right\}=\frac{\pi \beta}{2}$, we have from (2.21),

$$
\begin{aligned}
\operatorname{Arg}\left\{p\left(z_{0}\right)+\alpha\left(\frac{z p^{\prime}\left(z_{0}\right)}{p\left(z_{0}\right)}\right)\right\} & =\operatorname{Arg}\left\{p\left(z_{0}\right)\right\}+\operatorname{Arg}\left\{1+\alpha\left(\frac{z p^{\prime}\left(z_{0}\right)}{p^{2}\left(z_{0}\right)}\right)\right\} \\
& =\frac{\pi \beta}{2}+\operatorname{Arg}\left\{1+\frac{|\alpha| \beta k}{a^{\beta}} e^{-i \pi(1+\beta) / 2}\right\} \\
& \leq-\left\{\tan ^{-1}\left(\frac{|\alpha| \delta(\beta) \sin \frac{\pi(1+\beta)}{2}}{1+|\alpha| \delta(\beta) \cos \frac{\pi(1+\beta)}{2}}\right)-\frac{\pi \beta}{2}\right\} .
\end{aligned}
$$

This contradicts (2.19), and for the case $\operatorname{Arg}\left\{p\left(z_{0}\right)\right\}=-\frac{\pi \beta}{2}$, applying the same method as above, we have

$$
\operatorname{Arg}\left\{p\left(z_{0}\right)+\alpha\left(\frac{z p^{\prime}\left(z_{0}\right)}{p\left(z_{0}\right)}\right)\right\} \geq\left\{\tan ^{-1}\left(\frac{|\alpha| \delta(\beta) \sin \frac{\pi(1+\beta)}{2}}{1+|\alpha| \delta(\beta) \cos \frac{\pi(1+\beta)}{2}}\right)-\frac{\pi \beta}{2}\right\} .
$$

This contradicts also (2.19), and, therefore, it completes the proof. 
Theorem 2.11 Let $p(z)=1+\sum_{n=1}^{\infty} c_{n} z^{n}$ be analytic in the unit disc $\mathbb{D}$, and suppose that

$$
\begin{aligned}
& \left|\operatorname{Arg}\left\{p(z)+\alpha\left(\frac{z p^{\prime}(z)}{p(z)}\right)\right\}\right| \\
& \quad<\frac{\pi}{2}\left\{\beta+\frac{2}{\pi} \tan ^{-1} \frac{|\alpha| \delta(\beta) \sin ((1-\beta) \pi / 2)}{1+|\alpha| \delta(\beta) \cos ((1-\beta) \pi / 2)}\right\} \quad \text { for } z \in \mathbb{D},
\end{aligned}
$$

where $0<\alpha, 0<\beta<1$, and

$$
\delta(\beta)=\frac{\beta}{2}\left(\left(\frac{1-\beta}{1+\beta}\right)^{\beta+1}+\left(\frac{1-\beta}{1+\beta}\right)^{\beta-1}\right) \text { and } \alpha>\frac{\sin (\pi \beta / 2)}{\delta(\beta)}
$$

Then $\operatorname{Arg}\{p(z)\}<\frac{\pi \beta}{2}$ in $\mathbb{D}$.

Proof The proof runs as the previous proof, take $\alpha>0$ into account. Suppose that there exists a point $z_{0} \in \mathbb{D}$ such that

$$
|\operatorname{Arg}\{p(z)\}|<\frac{\pi \beta}{2} \quad \text { for }|z|<\left|z_{0}\right|
$$

and

$$
\left|\operatorname{Arg}\left\{p\left(z_{0}\right)\right\}\right|=\frac{\pi \beta}{2}
$$

then by Nunokawa's lemma [12], we have for the case $\operatorname{Arg}\left\{p\left(z_{0}\right)\right\}=\frac{\pi \beta}{2}$,

$$
\begin{aligned}
\operatorname{Arg}\left\{p\left(z_{0}\right)+\alpha\left(\frac{z p^{\prime}\left(z_{0}\right)}{p\left(z_{0}\right)}\right)\right\} & =\operatorname{Arg}\left\{p\left(z_{0}\right)\right\}+\operatorname{Arg}\left\{1+\alpha\left(\frac{z p^{\prime}\left(z_{0}\right)}{p^{2}\left(z_{0}\right)}\right)\right\} \\
& =\frac{\pi \beta}{2}+\operatorname{Arg}\left\{1+\frac{\alpha \beta k}{a^{\beta}} e^{i \pi(1+\beta) / 2}\right\} \\
& \leq \frac{\pi \beta}{2}+\tan ^{-1}\left(\frac{|\alpha| \delta(\beta) \sin \frac{\pi(1+\beta)}{2}}{1+|\alpha| \delta(\beta) \cos \frac{\pi(1+\beta)}{2}}\right) \frac{\pi \beta}{2}
\end{aligned}
$$

This contradicts (2.22), and for the case $\operatorname{Arg}\left\{p\left(z_{0}\right)\right\}=-\frac{\pi \beta}{2}$, applying the same method as above, we have

$$
\operatorname{Arg}\left\{p\left(z_{0}\right)+\alpha\left(\frac{z p^{\prime}\left(z_{0}\right)}{p\left(z_{0}\right)}\right)\right\} \leq-\left\{\frac{\pi \beta}{2}+\tan ^{-1}\left(\frac{|\alpha| \delta(\beta) \sin \frac{\pi(1+\beta)}{2}}{1+|\alpha| \delta(\beta) \cos \frac{\pi(1+\beta)}{2}}\right) \frac{\pi \beta}{2}\right\}
$$

This contradicts also (2.22), and, therefore, it completes the proof.

The authors declare that they have no competing interests. 


\section{Author details}

${ }^{1}$ University of Gunma, Hoshikuki-cho 798-8, Chuou-Ward, Chiba, 260-0808, Japan. ${ }^{2}$ Kinki University, Higashi-Osaka, Osaka, 577-8502, Japan. ${ }^{3}$ Pukyong National University, Pusan, 608-737, Korea. ${ }^{4}$ Department of Mathematics, Rzeszów University of Technology, Al. Powstańców Warszawy 12, Rzeszów, 35-959, Poland. ${ }^{5}$ Department of Mathematics and Computer Science, İstanbul Kültür University, İstanbul, Turkey.

\section{Acknowledgements}

This research was supported by the Basic Science Research Program through the National Research Foundation of Korea (NRF) funded by the Ministry of Education, Science and Technology (No. 2011-0007037).

Dedicated to Professor Hari M Srivastava.

Received: 4 April 2013 Accepted: 21 August 2013 Published: 8 September 2013

\section{References}

1. Stankiewicz, J: Quelques problèmes extrèmaux dans les classes des fonctions $\alpha$-angulairement ètoilèes. Ann. Univ. Mariae Curie-Skłodowska, Sect. A 20, 59-75 (1966)

2. Brannan, DA, Kirwan, WE: On some classes of bounded univalent functions. J. Lond. Math. Soc. 1(2), 431-443 (1969)

3. Nunokawa, M, Owa, S, Yavuz Duman, E, Aydoğan, M: Some properties of analytic functions relating to the Miller and Mocanu result. Comput. Math. Appl. 61, 1291-1295 (2011)

4. Mocanu, PT: Some starlikeness conditions for analytic functions. Rev. Roum. Math. Pures Appl. 33(1-2), 117-124 (1988)

5. Mocanu, PT: New extensions of the theorem of R. Singh and S. Singh. Mathematica 37(60), 171-182 (1995)

6. Aouf, MK, Dziok, J, Sokół, J: On a subclass of strongly starlike functions. Appl. Math. Lett. 24, $27-32$ (2011)

7. Sokół, J: On sufficient condition to be in a certain subclass of starlike functions defined by subordination. Appl. Math. Comput. 190, 237-241 (2007)

8. Sokół, J: On functions with derivative satisfying a geometric condition. Appl. Math. Comput. 204, 116-119 (2008)

9. Sokół, J: Coefficient estimates in a class of strongly starlike functions. Kyungpook Math. J. 49, 349-353 (2009)

10. Srivastava, HM: Generalized hypergeometric functions and associated families of $k$-starlike functions. Gen. Math. 15(2-3), 201-226 (2007)

11. Srivastava, HM, Lashin, AY: Subordination properties of certain classes of multivalently analytic functions. Math. Comput. Model. 52, 596-602 (2010)

12. Nunokawa, M: On the order of strongly starlikeness of strongly convex functions. Proc. Jpn. Acad., Ser. A, Math. Sci. 69(7), 234-237 (1993)

doi:10.1186/1029-242X-2013-426

Cite this article as: Nunokawa et al.: On the improvement of Mocanu's conditions. Journal of Inequalities and Applications 2013 2013:426.

\section{Submit your manuscript to a SpringerOpen ${ }^{\circ}$ journal and benefit from:}

- Convenient online submission

- Rigorous peer review

Immediate publication on acceptance

- Open access: articles freely available online

- High visibility within the field

- Retaining the copyright to your article 